\title{
Direct synthesis of hydrogenated graphene via hydrocarbon decomposition in plasmas
}

\author{
M. B. Shavelkina, R. H. Amirov \\ Joint Institute for High Temperatures of Russian Academy of Sciences, Izhorskaya st. 13 Bd. 2, Moscow, 125412 , \\ Russia \\ mshavelkina@gmail.com, amirovravil@yahoo.com
}

PACS 52.75.Hn, 52.77.Fv, 61.46.Fg, 68.37.Hk

DOI 10.17586/2220-8054-2019-10-1-102-106

\begin{abstract}
We study graphene synthesis in a plasma-jet reactor. Graphene is obtained via decomposition of hydrocarbons in the plasma produced in the DC plasma torch. The products of synthesis are characterized using the following methods: electron microscopy, Raman spectroscopy, X-ray diffraction and X-ray photoelectron spectroscopy, thermal analysis and express-gravimetry. We make the conclusion that, at the few-layer graphene samples, their hydrogenation takes place. The maximal hydrogen-to-carbon ratio was 1:4.
\end{abstract}

Keywords: Synthesis, graphene, graphane, plasma jet, hydrocarbons.

Received: 2 November 2018

Revised: 11 December 2018

\section{Introduction}

Graphene is a single layer of carbon atoms arranged in a hexagonal lattice and graphane is the hydrogenated graphene where each carbon atom is covalently connected with the hydrogen atom in the $\mathrm{CH}$ stoichiometry [1]. Here, the $\mathrm{sp}^{3}$-hybridized carbon atoms shift up or down from the planes; according to the calculations in [1], it is an energy-profitable system. The theoretically proposed models of the graphane structure are only partly confirmed theoretically [2]. In the conditions of wide investigations of potential graphane applications to the particular problems in various fields of technology, science, and industry, primarily for the hydrogen storage [1] or in precise devices [3] or solar batteries [4], the search for optimal graphene synthetic methods becomes especially urgent.

Recently, hydrogenated graphene structures were obtained, with the different hydrogen-to carbon stoichiometry depending on the production method. The methods based on application of low or high pressure the hydrogen containing gas medium result in lower sample saturation by hydrogen than the liquid-phase methods based on the modified Birch method [5-9]. Authors [10] used the scaled electrochemical approach to obtain the hydrogenated graphene from the solvated NR (4+) graphite. Yet, currently, totally hydrogenated graphene is still not synthesized. Even in order to obtain the graphene with various hydrogenation degree, at least two or more stages are required. At the first stage, the initial material, graphene, is obtained using one of the known methods: adhesive tape, CVD, Hummers' method, or on the $\mathrm{SiC}$ surface. At the second stage, the hydrogenation itself proceeds by means of physical or chemical methods. In liquid-phase approaches, an additional stage of refinement from the undesirable medium admixtures, i.e. atoms, molecules, small atomic clusters, is needed.

\section{Experimental setup and procedure}

Earlier, we investigated the possibility of synthesizing free graphene by means of high-power plasma torch at the pressure of $150-710$ Torr [11,12]. Analysis of the processes in the carbon nucleation zone of the plasma jet generated by the plasma torch indicates high concentration of the atomic hydrogen and of the $\mathrm{C}_{6} \mathrm{H}_{6}$-like compounds; these substances, according to [13-16], might promote the graphene formation. Additionally, it is common knowledge that application of the DC plasma torches makes it possible to synthesize high-quality pure carbon nanomaterials from inexpensive precursors [17], thus reducing the requirements to the carbon source, its cost and the final product cost. Authors [18-20] show that, with application of the plasma torches, high purity graphene might be obtained with controlled electronic properties.

The present work is aimed at experimental search to plasma-assisted synthesis of graphene with the attached hydrogen atoms. The plasma jets used here is the same one as in the work [21]. We performed decomposition of hydrocarbons in the thermal plasma jet up to the carbon vapor formation. Then we rapidly cooled the decomposition products of hydrocarbons in a vacuum chamber with the subsequent nucleation of solid carbon. We received synthesized products on the collector. Simultaneous input of the precursors (methane, propane-butane, acetylene) and the plasma-forming gas (helium) into the discharge gap of the plasma torch is an advantage of such method. 
Here, we might independently vary the component ratio in the plasma jet within wide ranges. Besides, the extending anode channel - the constructive peculiarity of the plasma torch - makes it possible to widely vary the rate of the vapor-gas flow cooling, to change its geometry, and, in general, to control the process efficiency [22].

Table 1 presents the parameters of experiments on the hydrogenated graphene synthesis. The synthesis process duration equals to $6 \mathrm{~min}$ and is governed by the quantity of specimens required to investigate their properties. Total output of the carbon sediment collected on the target varies from $6 \mathrm{~g}$ to $10 \mathrm{~g}(99 \mathrm{wt}$ \%).

TABLE 1. Experimental conditions

\begin{tabular}{|c|c|c|c|c|c|c|c|}
\hline $\begin{array}{l}\text { Power } \\
(\mathrm{kW})\end{array}$ & $\begin{array}{l}\text { Current } \\
\text { (A) }\end{array}$ & $\begin{array}{c}\text { Voltage } \\
\text { (V) }\end{array}$ & $\begin{array}{c}\text { Gas } \\
\text { pressure } \\
\text { (Torr) }\end{array}$ & $\begin{array}{l}\text { Helium } \\
\text { flow } \\
\text { rate } \\
\left(\mathrm{g} \cdot \mathrm{s}^{-1}\right)\end{array}$ & $\begin{array}{l}\text { Propane- } \\
\text { butane } \\
\text { flow } \\
\text { rate } \\
\left(\mathrm{g} \cdot \mathrm{s}^{-1}\right)\end{array}$ & $\begin{array}{l}\text { Methane } \\
\text { flow } \\
\text { rate } \\
\left(\mathrm{g} \cdot \mathrm{s}^{-1}\right)\end{array}$ & $\begin{array}{c}\text { Acetylene } \\
\text { flow } \\
\text { rate } \\
\left(\mathrm{g} \cdot \mathrm{s}^{-1}\right)\end{array}$ \\
\hline $22-36$ & $350-400$ & $60-90$ & $\begin{array}{l}150, \\
350, \\
710\end{array}$ & $0.75-0.9$ & $0.11-0.30$ & $0.15-0.37$ & $0.05-0.16$ \\
\hline
\end{tabular}

We measured the temperature in the zone of carbon vapor condensation and formation of graphene and graphane by the chromel-alumel thermocouple. Depending on the experimental conditions, the temperature on the collector surface was varied from $500-700{ }^{\circ} \mathrm{C}$. This temperature range is optimal to obtain the hydrogenated graphene [23].

The samples synthesized in the plasma jet volume were characterized by means of the standard methods of graphene materials investigation [7,24,25]. To provide the most accurate identification of the samples, we used a method of a scanning electron microscopy (SEM) with a Hitachi S5500 microscope in the DF-STEM, BF-STEM and SE modes and a Raman spectroscopy study (Nectra Spectra, a laser with a wavelength of $532 \mathrm{~nm}$ ). To quantitatively investigate the element composition and the electron state of the atoms, we used the X-ray photoelectron spectroscopy (XRPS) - the measurements were performed at the room temperature by means of the Theta Probe (ThermoFisher Scientific, GB) spectrometer: we took the Al K-a emission without using the system of the surface charging neutralization. To determine the element composition, we applied the vario MICRO cube express-gravimetry method. The phase composition of the synthesized samples was investigated by the thermal methods (thermogravimetry and differentially-scanning calorimetry), under linear heating with a rate of $10 \mathrm{~K} / \mathrm{min}$, under argon atmosphere, by means of the Netzsch (STA 409, PC Luxx) device. The X-ray structure analysis was performed using the standard technique and the DRON-2 facility (CuK $\alpha$-emission). We obtained the X-ray diffraction spectra from the basic surfaces of the samples.

\section{Results of experiments}

The electron microscopy results confirm the possibility of graphene hydrogenation at its synthesis. Fig. 1 shows the typical SEM images of the hydrogenated graphene structures obtained via decomposition of methane and acetylene in the helium plasma at 350 Torr and at the maximal consumption $(0.16-0.37 \mathrm{~g} / \mathrm{s}), \mathrm{Tab}$. 1 . In morphology, these structures are identical with the structures synthesized, with plasma application, in the experiments [20]. The samples represent the disoriented flakes, differing in the edge morphology and the lateral sizes (with sufficient scattering - from below $3 \mathrm{~nm}$ to above $1.5 \mu \mathrm{m}$ ). The X-ray analysis of the flakes shows that the spectrum of a typical sample contains two weak halos. The first of them is in the Bragg angle range $2 \theta=11-120$ and can belong to any hydrocarbon phase. The maximum intensity of this halo corresponds to the interplanar distance $d=0.7694 \mathrm{~nm}$. The second halo is located in the area of angles $2 \theta=24-280$. The maximum intensity of this halo corresponds to the interplanar spacing $d=0.3401 \mathrm{~nm}$. Study of morphology shows that the sample surface is wavy. Fig. 1 shows that the areas with swelling (covering not only the surface layer but also the next ones) rise above the waves. The swelling has a rhomboid shape which, as a rule, forms when a gas fills the layered structures. The rhomb angle equals to $120^{\circ}$ - typical for the crystal structure rather than for amorphous carbon. The swellings on the graphene surface are distributed irregularly. That very swelling effect was previously observed when studying the morphology of the pyrolytic graphite surface after the impact by the hydrogen flow [26].

The thermal sample analysis data concerning the hydrogen output are in agreement with the results obtained earlier in the works on the hydrogen desorption from both graphite [27] and graphene [24]. Fig. 2 shows an example of thermogram of the samples synthesized via the methane decomposition in the helium plasma at 

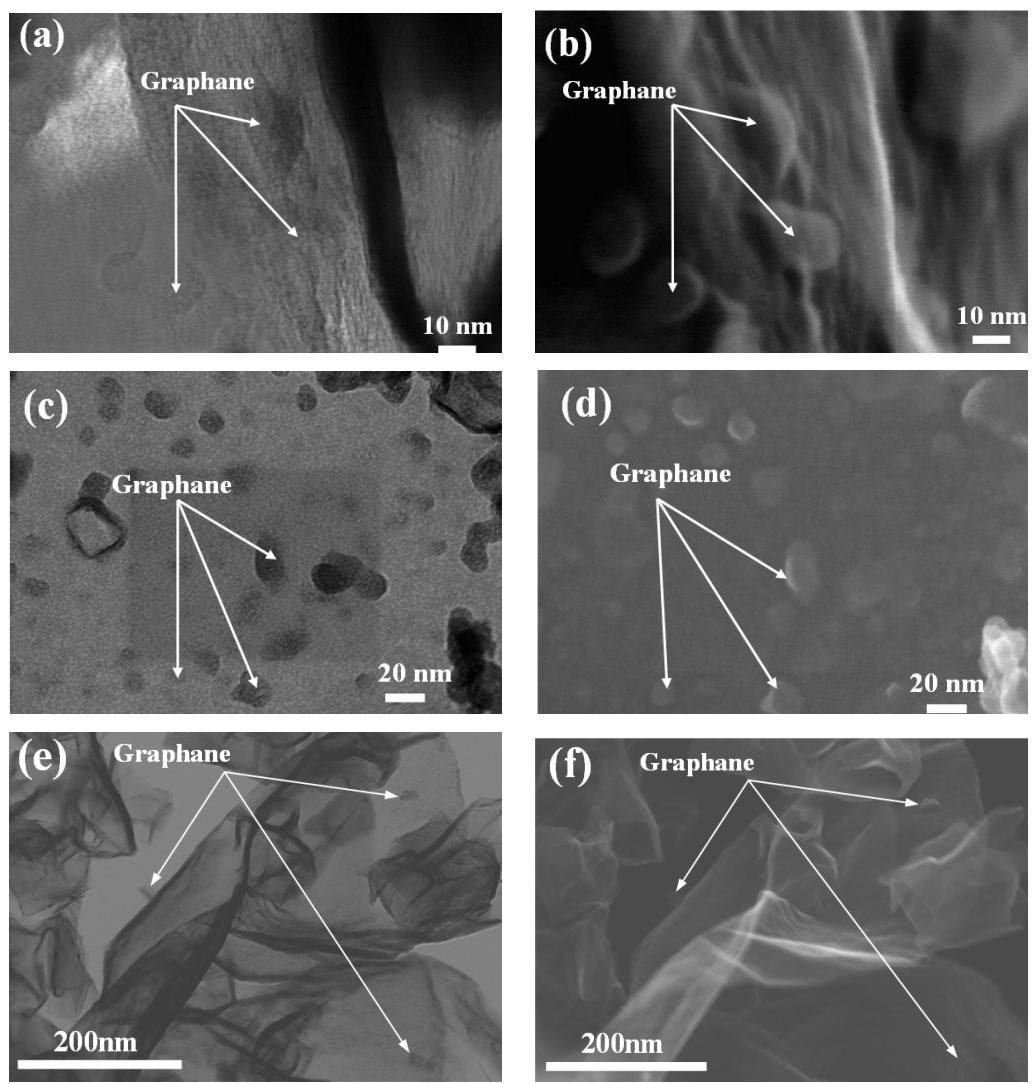

FIG. 1. (a) BF-STEM of the sample obtained via the propanebutane mixture decomposition in helium plasma at 350 Torr; (b) SEM of the sample obtained via the propanebutane mixture decomposition in helium plasma at 350 Torr; (c) SEM of the sample obtained via the acetylene decomposition in the helium plasma at 350 Torr; (d) BF-STEM of the sample obtained via the acetylene decomposition in helium plasma at 350 Torr; (e) SEM of the sample obtained via the methane decomposition in the helium plasma at 710 Torr; (f) BF-STEM of the sample obtained via the methane decomposition in helium plasma at 710 Torr

pressure of 710 Torr. Analysis of the thermogram shows that the content of the graphene component in the obtained hydrogenated material is above $60 \mathrm{wt}$ \% $\%$. Here, intensive mass decrease begins at $300{ }^{\circ} \mathrm{C}$ and continues up to above $1000{ }^{\circ} \mathrm{C}$. In the desorption curves, two desorption peaks are observed. Depending on the precursor type, the maximum falls on the temperature ranges of $300-400{ }^{\circ} \mathrm{C}$ and $800-900{ }^{\circ} \mathrm{C}$.

The XPS spectra also confirmed obtainment of graphane. Fig. 3 shows the high-resolution $\mathrm{C} 1 \mathrm{~s}$ spectra for the specimen obtained via the propane-butane mixture decomposition in the helium plasma at 710 Torr and the highly-oriented pyrolitic graphite (HOPG) measured at two angles against the normal to the surface. The spectra differ in the width of the main peak because of the specimen non-uniformity caused by the presence of the different size particles and the defects which is consistent with the work [28]. The difference in the spectra within the lower binding energy region indicates different conductivity caused, possibly, by the presence of the $\mathrm{C}-\mathrm{H}$ bonds - this fact is in agreement with results [24].

By means of the direct express-gravimetry method, we determine the C:H content ratio in the samples. The minimal quantity of the hydrogen atoms per a single carbon atom equals to 0.017 . This value was obtained when methane was used as the precursor. At the propane-butane mixture decomposition (with the flow rate of $0.37 \mathrm{~g} / \mathrm{s}$ ), in the helium plasma (710 Torr), with variation of the gas flow velocities, we obtained the hydrogen-to-carbon ratio of 1:4. The Raman-spectroscopic investigation of the samples also confirmed formation of graphane. Fig. 4 shows the Raman-spectrum of the samples synthesized from the propane-butane mixture in the helium plasma at different temperature on the collector surface [29]. These results respect to the Raman-spectrum of the single-layer graphene obtained via mechanical exfoliation (the adhesive tape) [24]. In Fig. 4, the D, G, and 2D peaks move up to $1337 \mathrm{~cm}^{-1}, 1569 \mathrm{~cm}^{-1}$, and $2659 \mathrm{~cm}^{-1}$, respectively. For all the spectra, the D line is present - due to the 


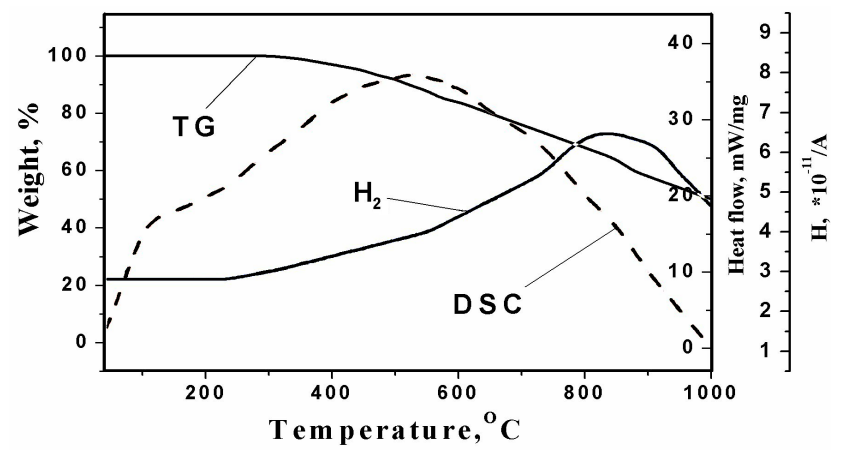

FIG. 2. Thermal analysis, in the inert medium, of the sample obtained via the methane decomposition in helium plasma at 710 Torr. The $\mathrm{G}$ is the sample mass variation, the DSC - variation of the sample heat release under the linear heating, $\mathrm{H}$ - the hydrogen output

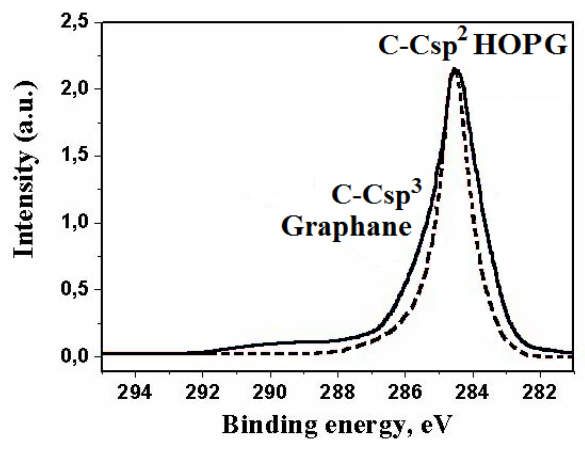

FIG. 3. Comparison of the C $1 \mathrm{~s}$ photoelectron spectra of the sample and of the HOPG measured at the various angles against the normal to the sample surface

morphology peculiarity of the structures, flakes with the fold edges. Certain discrepancy with the literary data is caused by application of the different lasers for excitement of the spectra - this fact is confirmed by the analysis in [30]. Figure 4 shows a typical spectrum of the hydrogenated graphene [31-34]. Note that the intensity shift of the breathing modes to the higher frequencies takes place; respectively, the $\mathrm{D}, \mathrm{G}$, and 2D peaks move up to $1350 \mathrm{~cm}^{-1}, 1580^{-1}$, and $2690 \mathrm{~cm}^{-1}$. Analysis of shape of the 2D peaks shows that the samples are two-layered. According to [35], the highest possibility of hydrogenation with respect to that, vary the bilayer graphene.

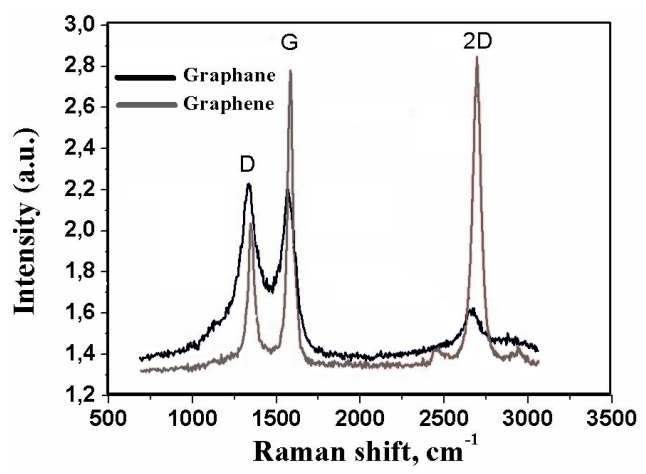

FIG. 4. Raman spectra of the samples obtained via the propanebutane mixture decomposition in helium plasma at 350 Torr

Measurements of the electrical conductivity of synthesized hydrogenated graphene from $10-50{ }^{\circ} \mathrm{C}$ using the classical four-probe method for bulk materials correlate with the data of [7] and correspond to the classical temperature dependence for a semiconductor. The band gap energy (equal to $0.22 \mathrm{eV}$ ) confirms their semiconductorlike character.

\section{Conclusions}

We demonstrate the possibility of synthesizing hydrogenated graphene structures in plasma jets. Their characterization by Raman spectroscopy, the XPS, the express-gravimetry and the thermal analysis methods shows that their properties are close to the properties of the hydrogenated single-layered graphene, both on the substrates and in the liquid media. We state the correlation between the degree of hydrogenation and the synthesis conditions. The hydrogen content depends on the synthesis conditions.

\section{Acknowledgements}

This work was financially supported by the Russian Fond of Basic Research (Grant No. 18-08-00040). 


\section{References}

[1] Sofo J.O., Chaudhari A.S., Barber G.D. Graphane: A two-dimensional hydrocarbon. Phys. Rev. B, 2007, 75, P. 153401.

[2] Chernozatonskii L.A., Artyukh A.A., Kvashnin D.G. Formation of graphene quantum dots by "Planting" hydrogen atoms at a graphene nanoribbon. JETP Lett., 2012, 95, P. 266-270.

[3] Huang L.F., Zeng Z. Lattice dynamics and disorder-induced contraction in functionalized graphene. J. Appl. Phys., 2013, 113, P. 083524.

[4] Gao H., Wang L., et al. Band gap tuning of hydrogenated graphene: H coverage and configuration dependence. J. Phys. Chem. C, 2011, 115, P. 3236-3242.

[5] Pumera M., Wong C.H. Graphane and hydrogenated graphene. Chem. Soc. Rev., 2013, 42, P. 5987-5995.

[6] Zhou C., Chen S., et al. Graphene's cousin: the present and future of graphane. Nanoscale Res. Lett., 2014,9 , P. 26.

[7] Jones J.D., Mahajan K.K., et al. Formation of graphane and partially hydrogenated graphene by electron irradiation of adsorbates on graphene. Carbon, 2010, 48, P. 2335-2340.

[8] Smith D., Howie R.T., et al. Hydrogenation of graphene by reaction at high pressure and high temperature. ACS Nano, 2015, 9, P. 82798283.

[9] Eng A.Y., Poh H.L., et al. Searching for magnetism in hydrogenated graphene: using highly hydrogenated graphene prepared via birch reduction of graphite oxides. ACS Nano, 2013, 7, P. 5930-5939.

[10] Abdelkader A.M., Patten H.V., et al. Electrochemical exfoliation of graphite in quaternary ammonium-based deep eutectic solvents: a route for the mass production of graphane. Nanoscale, 2015, 7, P. 11386-11392.

[11] Shavelkina M.B., Amirov R.H., Ivanov P.P., Filimonova E.A. Conversion of hydrocarbons in the plasma jet to produce carbon nanostructures. Proceedings of the Workshop on Magneto-Plasma Aerodynamics, Moscow, Russia, 5-7 April 2017, P. 92-93.

[12] Amirov R., Shavelkina M., et al. Direct synthesis of porous multilayer graphene materials using thermal plasma at low pressure. $J$. of Nanomaterials, 2015, 2015, P. 724508.

[13] Back M.H. Mechanism of the pyrolysis of acetylene. Can. J. of Chem., 1971, 49, P. 2199-2204.

[14] Tsyganov D., Bundaleska N., et al. On the plasma-based growth of 'flowing' graphene sheets at atmospheric pressure conditions. Plasma Sources Sci. Technol., 2016, 25, P. 015013.

[15] Norinaga K., Deutschmann O. Detailed kinetic modeling of gas-phase reactions in the chemical vapor deposition of carbon from light hydrocarbons. Ind. Eng. Chem. Res., 2007, 46, P. 3547-3557.

[16] Khalilov U., Bogaerts A., Neyts E.C. Microscopic mechanisms of vertical graphene and carbon nanotube cap nucleation from hydrocarbon growth precursors. Nanoscale, 2014, 6, P. 9206-9214.

[17] Keidar M., Shashurin A., et al. Arc plasma synthesis of carbon nanostructures: where is the frontier? J. Phys. D: Appl. Phys., 2011, 44, 174006.

[18] Wang Q., Wang X., Chai Z., Hu W. Low-temperature plasma synthesis of carbon nanotubes and graphene based materials and their fuel cell applications. Chem. Soc. Rev., 2013, 42, P. 8821-8834.

[19] Kim J., Suh J.S. Size-controllable and low-cost fabrication of graphene quantum dots using thermal plasma jet. ACS Nano, 2014, 8, P. 4190-4196.

[20] Kim J., Heo S.B., Gu G.H., Suh J.S. Fabrication of graphene flakes composed of multi-layer graphene sheets using a thermal plasma jet system. Nanotechnology, 2010, 21, P. 095601.

[21] Amirov R., Isakaev E., Shavelkina M., Shatalova T. Synthesis of carbon nanotubes by high current divergent anode-channel plasma torch. J. Phys.: Conf. Ser., 2014, 550, P. 012023.

[22] Isakaev E.Kh., Sinkevich O.A., et al. Thermal fluxes in a generator of low temperature plasma with a divergent channel of the outlet electrode. High Temperature, 2011, 6, P. 797-802.

[23] Tozzini V., Pellegrini V. Prospects for hydrogen storage in graphene. ArXiv:1207.5703, 2012. URL: https : //arxiv.org/abs/1207.5703.

[24] Elias D.C., Nair R.R, et al. Control of graphene's properties by reversible hydrogenation: evidence for graphane. Science, 2009, 323, P. 610-613.

[25] Sahin H., Leenaerts O., Singh S.K., Peeters F.M. Graphane. Wiley Interdiscip. Rev. Comput. Mol. Sci., 2015,5 , P. $255-272$.

[26] Scheffler M., Haberer D., et al. Probing local hydrogen impurities in quasi-free-standing graphene. ACS Nano, 2012, 6, P. 10590-10597.

[27] Denisov E.A., Kompaniets T.N., Kurdyumov A.A., Mazaev S.N. Atomic hydrogen interaction with various graphite types. J. Plasma Devices and Operations, 1998, 6, P. 265-269.

[28] Barinov A., Malcioglu O.B., et al. Initial Stages of Oxidation on Graphitic Surfaces: Photoemission Study and Density Functional Theory Calculations J. Phys. Chem. C, 2009, 113, . 9009-9013.

[29] Shavelkina M.B., Amirov R.H., Shatalova T.B. The effect of reactor geometry on the synthesis of graphene materials in plasma jets. J. of Phys.: Conf. Ser., 2017, 857, P. 012040.

[30] Ferrari A.C. Raman spectroscopy of graphene and graphite: disorder, electron-phonon coupling, doping and nonadiabatic effects. Solid State Commun., 2007, 143, P. 47-57.

[31] Ferrari A.C., Meyer C., et al. Raman spectrum of graphene and graphene layers. Phys. Rev. Lett., 2006,97, P. 187401.

[32] Jorio A., Dresselhaus M.S., Saito R., Dresselhaus G. Raman Spectroscopy in Graphene Related System, Wiley-VCH: Germany, 2011.

[33] Son J., Lee S., et al. Hydrogenated monolayer graphene with reversible and tunable wide band gap and its field-effect transistor. Nature Comm., 2016, 7, P. 13261.

[34] Cancado L.G., Jorio A., et al. Quantifying defects in graphene via raman spectroscopy at different excitation energies. Nano Lett., 2011, 11, P. 3190-3196.

[35] Nechaev Y.S., Veziroglu T.N. Thermodynamic aspects of the stability of the grapheme/graphane/hydrogen systems, relevance to the hydrogen on-board storage problem. Adv. Mat. Phys. Chem., 2013, 3, P. 255-280. 